\title{
Joint registration and super-resolution with omnidirectional images
}

\author{
Zafer Arican, Student Member, IEEE, and Pascal Frossard, Senior Member, IEEE
}

\begin{abstract}
This paper addresses the reconstruction of high resolution omnidirectional images from multiple low resolution images with inexact registration. When omnidirectional images from low resolution vision sensors can be uniquely mapped on the 2-sphere, such a reconstruction can be described as a transform domain super-resolution problem in the spherical imaging framework. We describe how several spherical images with arbitrary rotations in the $S O(3)$ rotation group contribute to the reconstruction of a high resolution image with help of the Spherical Fourier Transform (SFT). As low resolution images might not be perfectly registered in practice, the impact of inaccurate alignment on the transform coefficients is further analyzed. We then cast the joint registration and super-resolution problem as a total least squares norm minimization problem in the SFT domain. A $l_{1}$ - regularized total least squares problem is also considered. The regularized problem is solved efficiently by interior point methods. Experiments with synthetic and natural images show that the proposed scheme leads to effective reconstruction of high resolution images even when large registration errors exist in the low resolution images. The quality of the reconstructed images also increases rapidly with the number of low resolution images, which demonstrates the benefits of the proposed solution in super-resolution schemes. Finally, we highlight the benefit of the additional regularization constraint that clearly leads to reduced noise and improved reconstruction quality.
\end{abstract}

\section{INTRODUCTION}

$\mathbf{S}$ UPER-RESOLUTION typically describes the problem of the reconstruction of high quality images from multiple images of lower resolutions that are typically taken at different instant in times or from slightly different viewpoints. It permits to exploit images that are captured with low resolution sensors by exploiting efficiently their diversity in order to produce a high resolution image. Super-resolution has been quite an active field of research in different frameworks such as multi-view, video or multispectral imaging [1], [2], [3]. Efficient solutions to the super-resolution problem have been proposed with images from perspective cameras that are perfectly registered. As super-resolution is typically an illposed problem, some a priori information is usually exploited in the reconstruction of the high quality image through regularization methods. Regularization has been proved to be useful to increase the stability of ill-posed systems. Tikhonov and total variation (TV) algorithms are two common regularization methods using $\ell_{2}$ and $\ell_{1}$ norms respectively in order to improve the reconstruction performance.

Z. Arican is with the Signal Processing Laboratory (LTS4), Institute of Electrical Engineering, Ecole Polytechnique Fédérale de Lausanne (EPFL), Lausanne, 1015 Switzerland e-mail: zafer.arican@epfl.ch

P. Frossard is with the Signal Processing Laboratory (LTS4), Institute of Electrical Engineering, Ecole Polytechnique Fédérale de Lausanne (EPFL), Lausanne, 1015 Switzerland e-mail: pascal.frossard@epfl.ch.
While perfect registration has been a common assumption in most super-resolution works, it is quite rare in practice that the registration parameters can be obtained exactly, especially with low cost vision sensors. Small registration errors could be exploited to improve the reconstruction in some superresolution algorithms in low complexity solutions that avoid the use of regularization techniques [4]. However, it is usually preferable to solve the registration problem for better performance. Recent methods have therefore investigated the joint problem of the registration of low resolution images and superresolution reconstruction. For example, subspace methods and a projection theorem are used in [5] for estimating the registration parameters, followed by reconstruction of the high quality image. Other approaches have been proposed using respectively alternating minimization or structured nonlinear total least-squares norm with Gauss-Newton method in the pixel domain [6], [7]. Joint registration and super-resolution with $\ell_{1}$ regularization has been more recently proposed in [8].

None of the above methods can be easily adapted to omnidirectional images due to their specific geometry. Superresolution is however particularly interesting in the omnidirectional framework due to the particular design of most imaging systems that results in low sampling, and in particular low angular resolution. Omnidirectional images have the advantages to present a wider field of view than perspective camera images, but this often comes at a price of a lower resolution with most of the common sensors. However, the problem of super-resolution of omnidirectional images has not been widely studied. Algorithms used in super-resolution of perspective images have been applied to omnidirectional images [9], [10], but without exploiting the true geometry of the omnidirectional framework. The specific geometry of the problem has considered in super-resolution for spherical microphone arrays [11]. More recently, the specific characteristics of omnidirectional images have been considered in [12], where improved quality is obtained by an iterative projection solution with multiple images captured by pre-defined rotation of the camera around its main axis.

In this paper, we address the problem of joint registration and super-resolution of omnidirectional images that are captured with arbitrary rotation. As most omnidirectional images and in particular the images of catadioptric systems can be uniquely mapped onto the unit sphere [13], we propose to address the problem in a spherical framework. We build on our previous work [14], [15] and we propose a method that jointly estimates the registration errors and reconstructs high resolution images from low resolution spherical images with arbitrary rotations in the $\mathrm{SO}(3)$ rotation group. We propose to solve the super-resolution problem with help of the Spher- 
ical Fourier Transform (SFT) computed from non-uniformly sampled data on the sphere. We analyze the impact of the registration errors due to inaccurate rotation parameters on the coefficients of the SFT. This permits to cast the joint registration and super-resolution problem as a total least square minimization problem in the SFT domain. A $l_{1}$ regularization constraint is added to the minimization problem for further improvement again in the transform domain. The solution of the minimization problem by interior points methods permit to achieve efficient reconstruction even in the presence of large registration errors. Experiments with synthetic and natural images demonstrate the performance of the proposed solution, whose reconstruction quality gracefully improves with the number of low resolution images. In addition, the experimental results confirm the benefits of the regularization constraint the further improve the stability of the system and the quality of the reconstructed images.

The rest of the paper is organized as follows. Section II describes the spherical framework that is used in our transform domain super-resolution algorithm. The super-resolution problem with rotated images is described in Section III. Then Section IV presents the formulation of the joint registration and super-resolution problem and outlines the regularization constraints that are proposed for solving this ill-posed system. Finally, Section V presents experimental results that demonstrates the validity of the proposed algorithm for both synthetic and natural image sets.

\section{SPHERICAL IMAGING FRAMEWORK}

In this section, we introduce the spherical imaging framework and the notation that will be used in the paper. We derive the system that describe the problem of SFT-based superresolution on the sphere, and we study the influence of the registration error on the SFT coefficients.

First, we choose to work on the 2-sphere $S^{2}$, which is a natural spatial domain to perform processing of omnidirectional images as shown in [16] and references therein. For example, the images from catadioptric camera systems with a single effective viewpoint can be uniquely mapped onto a sphere via inverse stereographic projection [13], [17]. The centre of that sphere is co-located with the focal point of the parabolic mirror and each direction represents a light ray incident to that point. Therefore, we assume in this paper that the omnidirectional images are given as spherical images or that a pre-processing step transforms them into spherical images, as illustrated in Fig. 1. Note that this kind of transformation usually cannot guarantee an ideal sampling of the light information in practice due to the discretization imposed by the image sensor. But this is actually one of the motivations for super-resolution, as efficient reconstruction from multiple images could compensate the effects of performing the capture and discretization steps in different geometries.

The spherical image is formally denoted by $x(\theta, \phi)$, which belongs to the Hilbert space of square-integrable functions on the 2-sphere $S^{2}$. We assume that $x(\theta, \phi)$ is bandlimited to $B$. The parameters $\theta$ and $\phi$ correspond to the longitude and colatitude angles, which are in the range $[0, \pi]$ and $[-\pi, \pi)$,
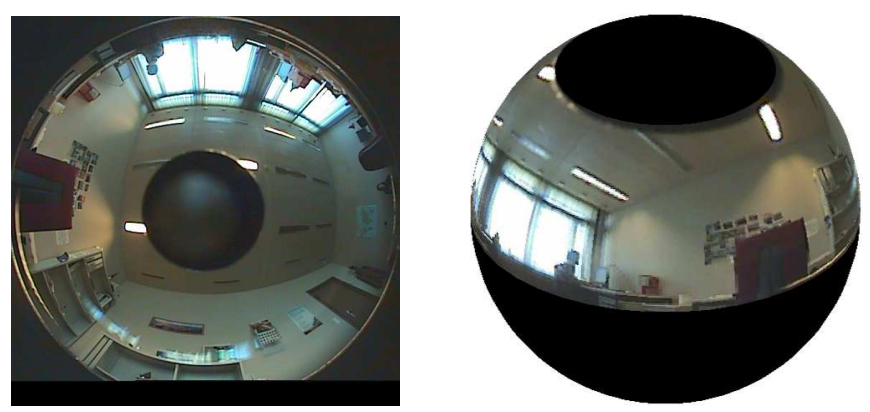

Fig. 1: Left: natural catadioptric image. Right: projection of the catadioptric image on the sphere.

respectively. These parameters form a $2 B \times 2 B$ equiangular grid on the sphere. We can obtain a transform representation of the image $x(\theta, \phi)$ with help of the Spherical Fourier Transform (SFT) [18] or its fast versions [19], [20]. The function $x(\theta, \phi)$ can be decomposed into a series of spherical harmonics $Y_{l}^{m}$ as

$$
x(\theta, \phi)=\sum_{l \in \mathbb{N}} \sum_{|m| \leq l} \hat{x}(l, m) Y_{l}^{m}(\theta, \phi)
$$

The Fourier coefficients $\hat{x}(l, m)$ are calculated as

$$
\hat{x}(l, m)=\int_{S^{2}} x(\theta, \phi) \bar{Y}_{l}^{m}(\theta, \phi) d \omega,
$$

where $d \omega=d \cos \theta d \phi$ is the rotation invariant Lebesque measure on the sphere and $\bar{Y}_{l}^{m}$ represent the spherical harmonics of order $(l, m)$. They are given by

$$
Y_{l}^{m}(\theta, \phi)=(-1)^{m} \sqrt{\frac{(2 l+1)(l-m) !}{4 \pi(l+m) !}} P_{l}^{m}(\cos \theta) e^{i m \phi},
$$

where $P_{l}^{m}(\cos \theta)$ are the associated Legendre functions (see Appendix). The interested readers are referred to [18] for more details on the construction of these functions.

As the signal is bandlimited, we have $\hat{x}(l, m)=0$ for $l \geq B$. Furthermore, the signal $x(\theta, \phi)$ can be perfectly reconstructed from uniformly sampled data on a $2 B \times 2 B$ equiangular grid.

When the sampling is irregular, one can still use a similar framework for the reconstruction of the bandlimited function $x(\theta, \phi)$ [21]. Let first denote by $P_{M}$ the space of polynomials on the sphere. These polynomials are given by :

$$
p(\theta, \phi)=\sum_{l=0}^{N-1} \sum_{|m| \leq l} a(l, m) Y_{l}^{m}(\theta, \phi) .
$$

The non-uniform samples on the unit sphere, $p_{j}\left(\theta_{j}, \phi_{j}\right)$ provide equations the represent the discretization of such polynomials on the sphere, which read

$$
p_{j}\left(\theta_{j}, \phi_{j}\right)=\sum_{l=0}^{N-1} \sum_{|m| \leq l} a(l, m) Y_{l}^{m}\left(\theta_{j}, \phi_{j}\right) .
$$

The set of equations represents a linear system that can be used for computing the coefficients $a(l, m)$, which generally provide an approximation of the SFT coefficients $\hat{x}(l, m)$. 
They can finally be substituted in Eq. (1) for the reconstruction of the function $x(\theta, \phi)$ on the unit sphere. The SFT framework is used for solving the super-resolution problem in the next section.

\section{SUPER-RESOLUTION WITH ROTATED IMAGES ON THE SPHERE}

The super-resolution problem is typically an inverse problem where a high resolution image $x$ is reconstructed from several images $z_{k}$ with lower resolution. The low resolution images can in general be modeled as

$$
z_{k}(\vartheta, \varphi)=\mathcal{D} \mathcal{T} x(\theta, \phi)+\epsilon_{k}(\vartheta, \varphi)
$$

where $\mathcal{D}$ and $\mathcal{T}$ are respectively downsampling and transformation operators and $\epsilon_{k}$ represents the approximation noise. We consider that we have $N$ low resolution signals that represent $L \times L$ spherical images, and we assume that the transformation operator $\mathcal{T}$ represents rotations in the rotation group $\mathrm{SO}(3)$. When all images live on a 2 -sphere, this operator permits to register the images in a common referential. Let $g_{k}=g_{Z Y Z}\left(\alpha_{k}, \beta_{k}, \gamma_{k}\right)$ denote a non-commutative rotation operator in the rotation group $S O(3)$. It describes the registration of the $k^{t h}$ low resolution image, which corresponds to the successive application of three rotations of angles $\alpha_{k}, \beta_{k}$, and $\gamma_{k}$ on the 2-sphere. The registration of the images produces an interlaced non-uniform sampling scheme as illustrated in Figure 2. After the mapping, the super-resolution problem becomes similar to the problem of reconstruction with nonuniformly sampled data on the sphere [21]. We describe in this section how the high resolution image can be approximated from low resolution rotated images with help of the Spherical Fourier Transform (SFT).

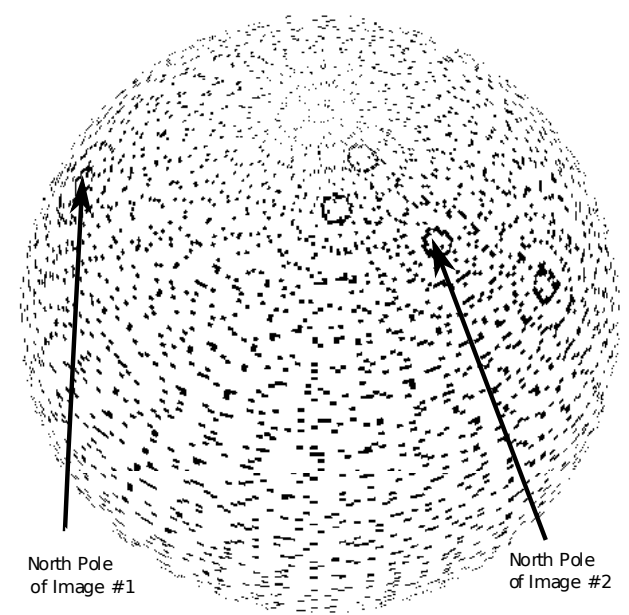

Fig. 2: Non-uniform sampling grid formed by low resolution images with different orientations.

We first provide a registration model that permits to describe the effect of rotation on the SFT representation of the images. When an image is transformed by a rotation operator in $\mathrm{SO}(3)$, the spherical harmonics after rotation can be expressed as a function of the spherical harmonics before rotation [22]. In particular, if the rotation operator $g$ defines a rotation with angles $(\alpha, \beta, \gamma)$ that maps the point $(\theta, \phi)$ to the point $\left(\theta^{\prime}, \phi^{\prime}\right)$ on the 2-sphere, we can write:

$$
Y_{l}^{n}\left(\theta^{\prime}, \phi^{\prime}\right)=\sum_{m=-l}^{l} U_{m n}^{l}(g) Y_{l}^{n}(\theta, \phi)
$$

where $U_{m n}^{l}(g)$ is an operator given by

$$
U_{m n}^{l}(g)=e^{i m \alpha} P_{m n}^{l}(\cos \beta) e^{i n \gamma} .
$$

The function $P_{m n}^{l}(\cos \beta)$ is the generalization of the associated Legendre polynomials. The main properties of these polynomials are given in Appendix A, along with fast calculation methods. We can note that $U_{m n}^{l}(g)$ is independent of the angular position of the sampling point.

The interesting property in Eq. (7) permits to represent multiple images with different rotations in $\mathrm{SO}(3)$ in the same transform domain. We can therefore compute the SFT coefficients of a high resolution image from samples of multiple low resolution images that are properly registered. The super-resolution problem becomes equivalent to the problem of reconstruction from samples arbitrarily distributed on the sphere [21], whose solution is similar to the method described in the previous section.

In particular, it is possible to rewrite Eq. (7) in matrix form. First we can write, for each spherical harmonics of degree $l$,

$$
\mathbf{Y}^{l}\left(\theta^{\prime}, \phi^{\prime}\right)=\mathbf{U}^{l}(g) \mathbf{Y}^{l}(\theta, \phi)
$$

where $\mathbf{U}^{l}(g)$ is a $(2 l+1) \times(2 l+1)$ matrix whose elements are given for $U_{m n}^{l}(g), \forall m, n$ such that $-l \leq m, n \leq l$. Then, if we gather all the spherical harmonics of degree $l$ in the same representation (with $l=0 \ldots(B-1)$ ), we have

$$
\mathbf{Y}\left(\theta^{\prime}, \phi^{\prime}\right)=\mathbf{U}(g) \mathbf{Y}(\theta, \phi) .
$$

The matrix $\mathbf{U}(g)$ is here a $B^{2} \times B^{2}$ block diagonal matrix of the form

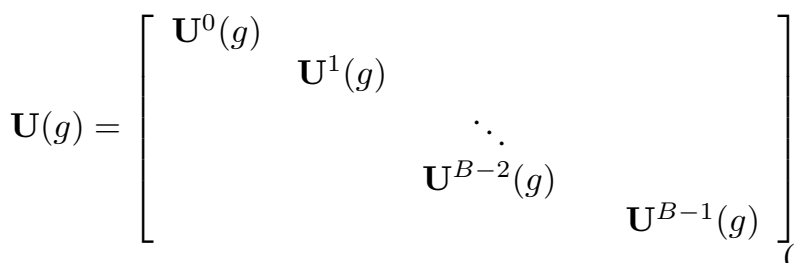

We can now gather the $N$ low resolution images in the same system. We first characterize the sensor or the sampling scheme in the sensing device by the grid $G_{0}$, which represents the set of positions on the 2-sphere where light intensity is recorded. The set of spherical harmonics corresponding to this grid is given by $\mathbf{Y}_{0}$, where $\mathbf{Y}_{0}=\left\{\mathbf{Y}(\theta, \phi):(\theta, \phi) \in G_{0}\right\}$.

The set of spherical harmonics $\mathbf{Y}_{k}$ of the $k^{t h}$ low resolution image can be seen as the result of a sampling with a grid $G_{k}$ that is a rotated version of the grid $G_{0}$ by the action of the rotation operator $g_{k}$. From the above properties, we can write:

$$
\mathbf{Y}_{k}^{\mathrm{T}}=\mathbf{U}\left(g_{k}\right) \mathbf{Y}_{0}^{\mathrm{T}}
$$

Finally, we can gather the sets of spherical harmonics given by the different sensor orientations in the same matrix. By writing $\mathbf{U}_{k}=\mathbf{U}\left(g_{k}\right)$, we have 


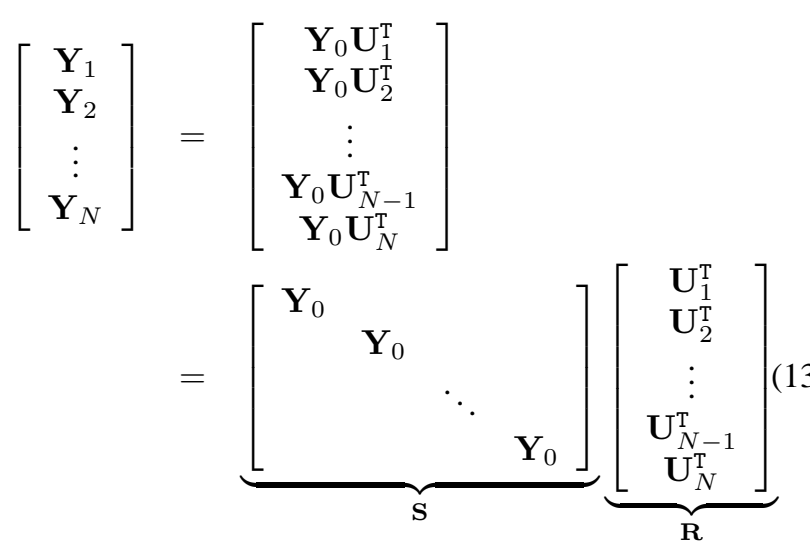

The matrix $\mathbf{S}$ depends on the sampling done by the sensor. The matrix $\mathbf{R}$ is a function of the rotations and models the registration of the low resolution images on the sphere. The linear system of Eq. (13) can be used to reconstruct the full resolution image, as explained in the previous section. We can estimate the Fourier coefficients of the high resolution image from the samples of the low resolution images after proper registration. These samples form a non-uniformly sampled grid on the sphere, and the Fourier coefficients can be estimated as the solution an inverse problem [21]. We have the following linear system

$$
\mathbf{S} \mathbf{R} \mathbf{a}=\mathbf{z},
$$

where $\mathbf{a}$ is the vector of Fourier coefficients and $\mathbf{z}$ is the set of samples from the low resolution images. One can estimate the Fourier coefficients $\hat{x}(l, m) \approx a(l, m)$ by solving the above system with least-square minimization methods for example. The high resolution image $x(\theta, \phi)$ can then be reconstructed by inverse spherical Fourier transform.

We have assumed in this section that the images can be perfectly registered, which is unfortunately not the case in practice. We note however that we can separate the effect of sampling and the influence of the registration of the images in the system of Eq. (14). This interesting property is exploited in the next section for the joint registration and super-resolution problem.

\section{JOINT REGISTRATION AND SUPER-RESOLUTION}

\section{A. Structured total least square minimization problem}

We have seen in the previous section that the high resolution image can be reconstructed from samples of multiple low resolution images after registration. However, when the images are not perfectly registered, some noise is introduced in the system of Eq. (14). Total least squares methods could be used to solve this kind of noisy systems, but such methods usually do not consider any particular structure in the system matrix. We have seen however that our system matrix $\mathbf{S} \mathbf{R}$ has an interesting structure that permits to separate the effect of registration due to the properties of spherical harmonics. We can therefore use structured total least squares minimization methods to estimate the Fourier coefficients from imperfectly registered images and later reconstruct the high resolution image.
Our data matrix $\mathbf{S} \mathbf{R}$ is the product of a sensor-specific sampling matrix and a registration matrix. The registration matrix $\mathbf{R}$ is a function of the rotations parameters for the multiple low resolution images. We denote by $\mathbf{g}$ the vector of the rotation angles $g_{k}=\left\{\alpha_{k}, \beta_{k}, \gamma_{k}\right\}$ that represent the rotation of the $k^{t h}$ low-resolution input image. When the images are not perfectly registered, the rotation vector $\mathbf{g}$ is unknown or known approximately. The joint registration and super-resolution problem can thus be written as follows.

Problem 1: Estimate jointly the Fourier coefficients $\mathbf{a}^{*}$ and the rotation vector $\mathbf{g}^{*}$ such that:

$$
\left\{\mathbf{a}^{*}, \mathbf{g}^{*}\right\}=\underset{\mathbf{a}, \mathbf{g}}{\operatorname{argmin}}\|\mathbf{z}-\mathbf{S} \mathbf{R}(\mathbf{g}) \mathbf{a}\|_{2}
$$

This is a structured total least square minimization problem. We denote by $\Delta \mathbf{a}$ a small change in $\mathbf{a}$ and by $\Delta \mathrm{g}$ a small change in g. The above problem can be approximated by linearization. We first have

$\mathbf{S ~ R}(\mathbf{g}+\Delta \mathbf{g})(\mathbf{a}+\Delta \mathbf{a}) \approx \mathbf{S ~ R}(\mathbf{g}) \mathbf{a}+\mathbf{J}(\mathbf{a}, \mathbf{g}) \Delta \mathbf{g}+\mathbf{S ~ R}(\mathbf{g}) \Delta \mathbf{a}$

The term $\mathbf{J}(\mathbf{a}, \mathbf{g})$ represents the Jacobian of $\mathbf{S R}(\mathbf{g})$ with respect to $\mathrm{g}$. It is written as

$$
\begin{aligned}
\mathbf{J}(\mathbf{a}, \mathbf{g}) & =\frac{\partial(\mathbf{S ~ R}(\mathbf{g}) \mathbf{a})}{\partial \mathbf{g}} \\
& =S \frac{\partial \mathbf{R}(\mathbf{g})}{\partial \mathbf{g}} \mathbf{a}
\end{aligned}
$$

Furthermore, since the rotation of each image is independent, $\frac{\partial \mathbf{R}(\mathrm{g})}{\partial \mathbf{g}}$ has the form

$$
\frac{\partial \mathbf{R}(\mathbf{g})}{\partial \mathbf{g}}=\left[\begin{array}{ccc}
\frac{\partial \mathbf{U}_{1}^{\mathrm{T}}\left(\mathbf{g}_{1}\right)}{\partial \mathbf{g}_{1}} & \ldots & \mathbf{0} \\
\vdots & \ddots & \vdots \\
\mathbf{0} & \ldots & \frac{\partial \mathbf{U}_{N}^{\mathrm{T}}\left(\mathbf{g}_{\mathrm{N}}\right)}{\partial \mathbf{g}_{\mathrm{N}}}
\end{array}\right]
$$

With this linearization, the Problem 1 can be solved iteratively, where each iteration has to solve a minimization problem of the following form

$\underset{\Delta \mathbf{a}, \Delta \mathbf{g}}{\operatorname{argmin}}\left\|\left(\begin{array}{cc}\mathbf{J}(\mathbf{a}, \mathbf{g}) & \mathbf{S ~ R}(\mathbf{g}) \\ \mathbf{L} & \mathbf{0}\end{array}\right)\left(\begin{array}{c}\Delta \mathbf{g} \\ \Delta \mathbf{a}\end{array}\right)+\left(\begin{array}{c}-\mathbf{z}+\mathbf{S} \mathbf{R}(\mathbf{g}) \mathbf{a} \\ \mathbf{0}\end{array}\right)\right\|_{2}$

The extra term $\mathbf{L} . \mathbf{L}=\sqrt{c} \mathbf{I}$ is a regularization term that increases the stability of the system in case $\mathbf{J}(\mathbf{a}, \mathbf{b})$ is close to zero. The parameters $\left\{\mathbf{a}^{*}, \mathbf{g}^{*}\right\}$ can be determined iteratively by finding incremental changes to the coefficients and rotation vectors that successively decrease the total least square norm. Due to the structure of the matrix in Eq. (19) the minimization turns into a Levenberg-Marquardt minimization algorithm with the parameter $c$ equal to Marquardt parameter. The overall algorithm steps therefore similar to the Levenberg-Marquardt method [23].

While we have provided a general form of the structured total least squares minimization problem for joint registration and super-resolution, the registration is generally defined with respect to one of the low resolution images, which is taken as a reference. We typically chose the first image as a reference, and the rotation of all the other images is then defined relatively to the reference image. The number of unknowns 
in the rotation vector becomes therefore smaller in practice. The adaptation of the above solution is straightforward.

Finally, we note that the joint registration and superresolution problem has been addressed previously in [7]. A Levenberg-Marquardt algorithm has been proposed to solve a least square minimization problem with additional Tikhonov regularization. However, as the computation has to be done in the pixel domain, the computation of Jacobian matrices is quite complex. As our method works in the Fourier domain, we can better separate the problems of coefficient approximation and registration estimation, which leads to a simpler solution.

\section{B. $\ell_{1}$-regularized problem}

The joint registration and super-resolution problem is typically an ill-posed problem. There are multiple solutions to the above problem, and the minimization of the total least squares norm does not guarantee to lead to the best solution in terms of image quality. In particular, the Problem 1 does not put any constraint on the SFT coefficients, so that the solution may present a lot of small coefficients that actually resembles more to noise than to actual image information.

We propose here an extension of the previous method by adding a $\ell_{1}$ regularization term on the SFT coefficients. The spherical harmonics typically have high peaks at different positions of the spectrum. The minimization of the $\ell_{1}$ norm of the Fourier coefficient vector helps to preserve the high values in the spectrum, and to smoothen out the low coefficients that mostly describe noise. The minimization problem with $\ell_{1}$ regularization take the following form.

Problem 2: Estimate jointly the Fourier coefficients $\mathbf{a}^{*}$ and the rotation vector $\mathrm{g}^{*}$ such that:

$$
\left\{\mathbf{a}^{*}, \mathbf{g}^{*}\right\}=\underset{\mathbf{a}, \mathbf{g}}{\operatorname{argmin}}\left[\|\mathbf{S} \mathbf{R}(\mathbf{g}) \mathbf{a}-\mathbf{z}\|_{2}^{2}+\lambda\|\mathbf{a}\|_{1}\right] .
$$

As the regularization term is not differentiable, the Problem 2 cannot be solved with a Levenberg-Marquardt minimization algorithm or a Newton-based methods. As proposed in [24], the cost function in the minimization problem of Eq. (20) can be converted into

$$
\|\mathbf{S} \mathbf{R}(\mathbf{g}) \mathbf{a}-\mathbf{z}\|_{2}^{2}+\lambda \sum_{i} u_{i}
$$

with $-u_{i}<a_{i}<u_{i}$ and $u_{i}>0$. The inequality constraint can actually be added into the cost function via a logarithmic barrier function to form the following cost function

$$
\|\mathbf{S} \mathbf{R}(\mathbf{g}) \mathbf{a}-\mathbf{z}\|_{2}^{2}+\lambda \sum_{i} u_{i}+t \phi(\mathbf{u}, \mathbf{a})
$$

The barrier function $\phi(.,$.$) for complex variables is selected$ as

$$
\phi(\mathbf{u}, \mathbf{a})=-\sum_{i} \log \left(u_{i}^{2}-\operatorname{Re}\left(a_{i}\right)^{2}-\operatorname{Im}\left(a_{i}\right)^{2}\right),
$$

which leads to a cost function that is now differentiable. As the other elements of the system have to be real-valued too, we transform the complex valued matrices and vectors into real-valued ones, similarly to [24]. In particular, a complex matrix $A$ and a complex vector $z$ are respectively converted into

$$
\begin{aligned}
\widetilde{\mathbf{A}} & =\left[\begin{array}{cc}
\operatorname{Re}(A) & -\operatorname{Im}(A) \\
\operatorname{Im}(A) & \operatorname{Re}(A)
\end{array}\right] \\
\widetilde{\mathbf{z}} & =\left[\begin{array}{l}
\operatorname{Re}(z) \\
\operatorname{Im}(z)
\end{array}\right]
\end{aligned}
$$

We can again approximate the first part of the cost function by linearization as a function of $\Delta \mathbf{a}$ and $\Delta \mathrm{g}$. Then we can iteratively solve the Problem 2, where the search direction at each iteration is given by

$$
\mathbf{H}\left[\begin{array}{c}
\Delta \mathbf{g} \\
\Delta \widetilde{\mathbf{a}} \\
\Delta \mathbf{u}
\end{array}\right]=-\nabla
$$

where $\mathbf{H}$ is the Hessian matrix of the system and $\nabla$ is the gradient.

The computation of the Hessian matrix is however quite complex for the registration parameters. We propose to approximate the components that correspond to the registration parameters by first-order derivatives in $\mathbf{H}$. We thus have

$$
\mathbf{H} \approx\left[\begin{array}{ccc}
\widetilde{\mathbf{J}}^{\mathrm{T}} \widetilde{\mathbf{J}}+c \mathbf{I} & \widetilde{\mathbf{J}}^{\mathrm{T}} \widetilde{\mathbf{S}} \widetilde{\mathbf{R}}(\mathbf{g}) & \mathbf{0} \\
\widetilde{\mathbf{R}}^{\mathrm{T}}(\mathbf{g}) \widetilde{\mathbf{S}}^{\mathrm{T}} \widetilde{\mathbf{J}} & \mathbf{D}_{1} & \mathbf{D}_{2} \\
\mathbf{0} & \mathbf{D}_{3} & \mathbf{D}_{4}
\end{array}\right]
$$

where $\widetilde{\mathbf{J}}$ is the Jacobian of the cost function with respect to g. The term

$$
\left[\begin{array}{ll}
\mathbf{D}_{1} & \mathbf{D}_{2} \\
\mathbf{D}_{3} & \mathbf{D}_{4}
\end{array}\right]
$$

represents the Hessian with respect to the $\tilde{\mathbf{a}}$ and $\tilde{\mathbf{u}}$.

Finally, at each iteration of the algorithm, the unknown vectors $\mathbf{b}, \mathbf{a}$ and $\mathbf{u}$ are updated by solving the system of Eq. (25). The unknown vectors are updated with the values of the step direction given by $\Delta \mathrm{g}, \Delta \mathrm{a}$, and $\Delta \mathrm{u}$. The algorithm proceeds until a maximum number of iterations is reached, or until the decrease of the cost function at each iteration becomes negligible.

\section{EXPERIMENTAL RESULTS}

\section{A. Total least square minimization algorithm}

We analyze in this section the performance of the joint registration and super-resolution algorithms proposed in the previous section. We perform experiments on both synthetic data and natural images. We analyze the performance of the proposed algorithms with respect to the number of low resolution images. We study also their robustness to noise in the low resolution images. We also look at the influence of the system design parameters, such as the sampling scheme of the sensor.

We first analyze the performance of the LevenbergMarquardt algorithm of Section IV-A with synthetic spherical images of realistic looking room scene. We first reconstruct a $128 \times 128$ image from 80 low resolution images of $16 \times 16$ pixels. The rotation angles for the low-resolution images are randomly selected with a uniform distribution. A random registration error of maximum 5 degrees with a uniform distribution is further introduced for each rotation angle. We 


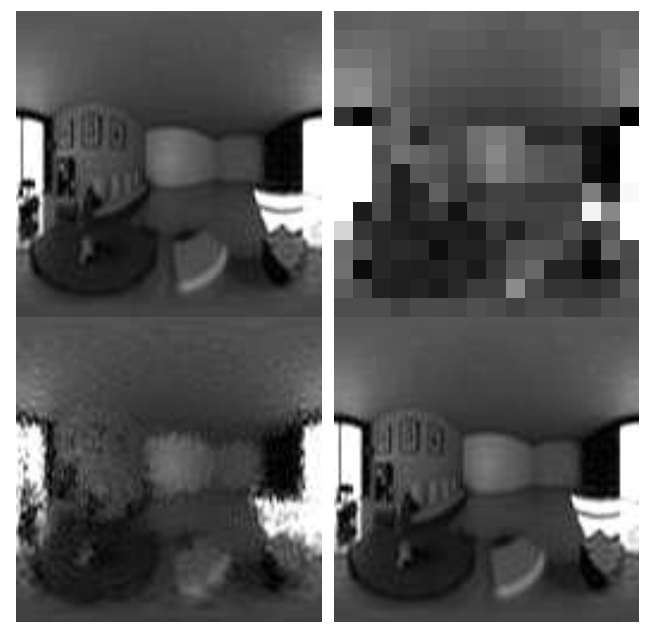

Fig. 3: Groundtruth high-resolution image (top-left), one lowresolution image (top-right), reconstructed image with registration errors, PSNR $=24.30 \mathrm{~dB}$ (bottom-left), reconstructed image with the Levenberg-Marquardt algorithm. PSNR = $44.28 \mathrm{~dB}$. (bottom-right).
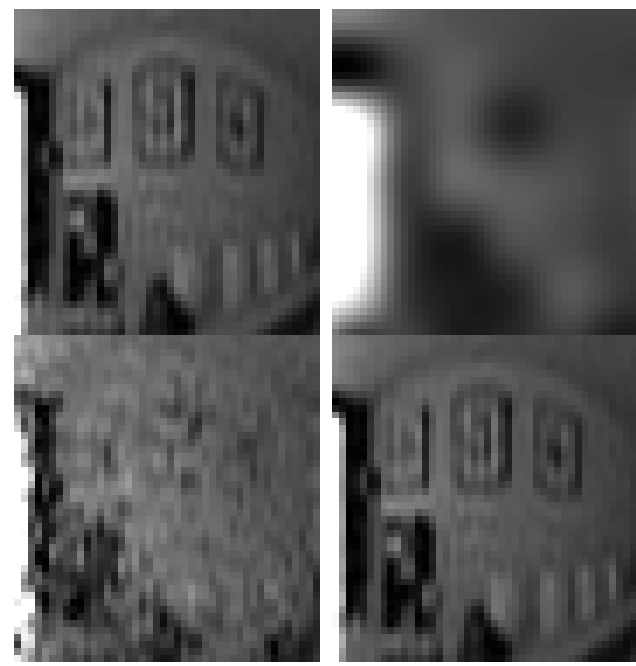

Fig. 4: Zoom in the synthetic images. Groundtruth highresolution image (top-left), one low-resolution image (topright), reconstructed image with registration errors (bottomleft), reconstructed image with the Levenberg-Marquardt algorithm (bottom-right).

compare the reconstruction of a high resolution image without correction of the registration error (given by solving Eq. (14)) and respectively with joint registration and super-resolution (as given in Problem 1). Figure 3 shows the reconstruction of the high resolution image, while Figure 4 proposes a zoom on a highly textured region in the image. We see that the registration errors highly affect the reconstructed image when the registration is not corrected. The structured total least squares minimization methods that jointly performs registration and reconstruction is able to correct these registration errors and provides an effective approximation of the high resolution image.

We now observe the effect of number of low-resolution

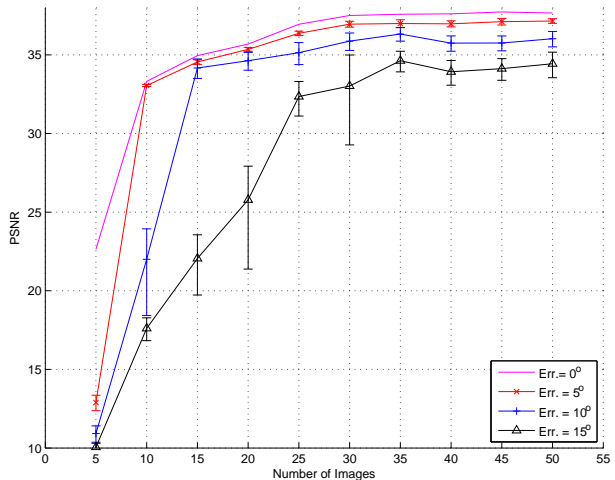

Fig. 5: Reconstruction quality of a 64 x 64 pixels omnidirectional image as a function of the number of low resolution images (16 x 16 pixels), for the Levenberg-Marquardt algorithm.

images on the convergence of the proposed algorithm for different registration errors. We use $16 \times 16$ low-resolution images to reconstruct a $64 \times 64$ high-resolution image. We randomly generate registration errors of maximum 5,10 and 15 degrees with a uniform distribution with zero mean. The Figure 5 shows the PSNR values for the reconstructed images with the Levenberg-Marquardt algorithm for different number of images and registration errors. We see that for small registration errors, an accurate high-resolution image can be generated from a small number of low resolution images. The number of images required for a good approximation of the high resolution image augments with the registration errors, as expected.

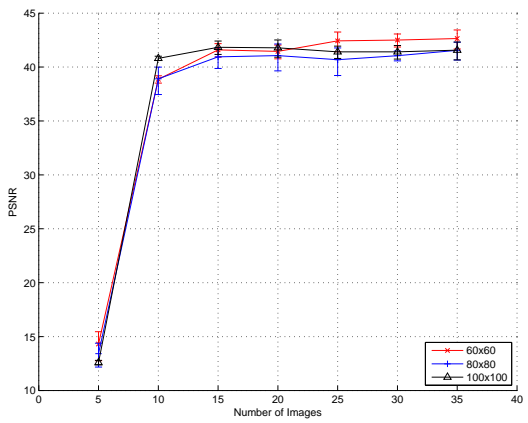

Fig. 6: Reconstruction quality for the Levenberg-Marquardt algorithm for images of different resolutions.

We then observe the influence of resolution of the low resolution images on the convergence of the Levenberg-Marquardt algorithm and the quality of the reconstructed image. We approximate images of size $60 \times 60,80 \times 80$ and $100 \times 100$ from multiple low resolution images of $15 \times 15,20 \times 20$ and $25 \times 25$ respectively. We again consider a random registration noise of at most 5 degrees with a uniform distribution. Figure 6 shows that the joint registration and super-resolution algorithm is not really affected by the resolution of the images. The algorithm converges to similar reconstruction qualities for all resolutions.

Then, we look at the robustness of the joint registration and super-resolution algorithm in the case where the low 


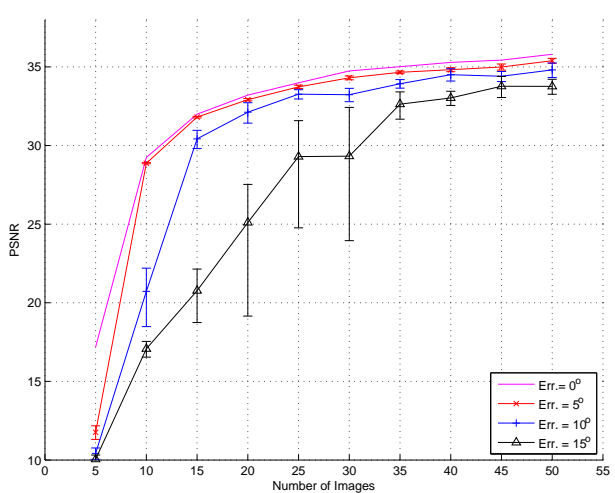

Fig. 7: The reconstruction quality on existence of image noise of $\mathrm{SNR}=30 \mathrm{~dB}$

resolution images are affected by noise. We use $16 \times 16$ lowresolution images to reconstruct a $64 \times 64$ high-resolution image. We randomly generate registration errors of maximum 5,10 and 15 degrees with a uniform distribution with zero mean. We further add a white gaussian noise with zero mean to the low resolution images, which results into a SNR of $30 \mathrm{~dB}$. The Levenberg-Marquardt algorithm is then used for the reconstruction of the high resolution image. Figure 7 shows the reconstruction quality for different registration errors and noisy images. We see that the reconstruction algorithm demonstrates similar performance as in the noiseless case especially when the number of images is high enough. The algorithm appears to be quite robust to additive noise even if the quality unsurprisingly stays lower than the noiseless case.

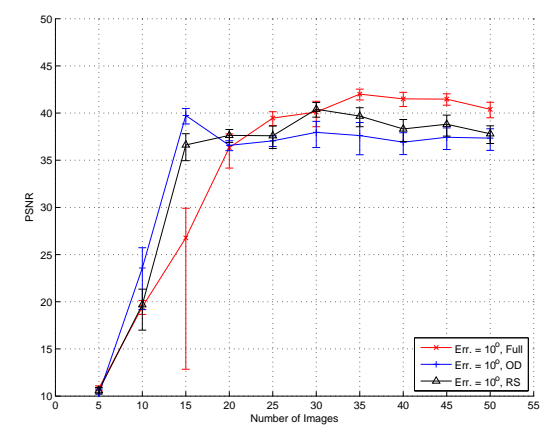

Fig. 8: Reconstruction quality vs number of low resolution images for an equiangular sampling (Full), a catadioptric grid (OD) and a random sampling (RS).

Finally, we test three different sampling schemes in the generation of the low resolution images. As shown in Section III, the Levenberg-Marquardt algorithm is independent of the sampling structure of the sensor. We reconstruct a $64 \times 64$ spherical image from multiple low resolution images of 256 samples each, but where the samples are given following three different structures: (i) a 16x16 equiangular grid, (ii) a 9x28 sampling grid corresponding to a catadioptric system and (iii) a random grid of 256 samples with a uniform distribution on sphere. A random registration error of at most 10 degrees with uniform distribution is applied to each image. Figure 8 shows the quality of the reconstructed images as a function of the number of low resolution images, for different sampling schemes. All three sampling schemes result into the same image quality and all nicely converge when the number of images increases. Fig 9 illustrates the resulting reconstructed images generated from 50 images for each of the sampling grid.
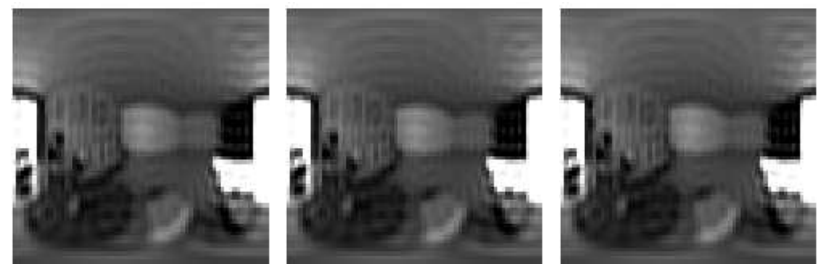

Fig. 9: Reconstructed image with 50 images for an equiangular grid, catadioptric grid and random sampling scheme (from left to right).

\section{B. Performance of the regularized solution}

We study now the performance of the regularized solution to the joint registration and super-resolution problem. We compared the results of the Levenberg-Marquardt algorithm with the results of the $\ell_{1}$-regularization proposed in Problem 2. For the sake of completeness, we also show the performance of an $\ell_{2}$-regularization method from [24], which we have adapted to our problem. In particular, the $\ell_{2}$-regularized problem takes the following form

$$
\left\{\mathbf{a}^{*}, \mathbf{g}^{*}\right\}=\underset{\mathbf{a}, \mathbf{g}}{\operatorname{argmin}}\left[\|\mathbf{S} \mathbf{R}(\mathbf{g}) \mathbf{a}-\mathbf{z}\|_{2}^{2}+\lambda\|\mathbf{a}\|_{2}^{2}\right] .
$$

This is a nonlinear system due to the registration vector $\mathbf{g}$, but all terms can still be differentiated so that the problem can be solved with Newton-based methods. We compare the different regularization strategies for the reconstruction of $64 \times 64$ pixel images from $16 \times 16$ pixel images. We further consider the cases where the low resolution images respectively have no registration error, and random registration errors of maximum 10 degrees with a uniform distribution. Figure 10 shows the influence of $\ell_{1}$ and $\ell_{2}$ regularization terms on the quality of the reconstructed image as a function of the number of low resolution images. Both regularization methods improve the quality compared to the Levenberg-Marquardt algorithm. However, the $\ell_{1}$-regularization provides better performance since it manages to preserve the peaks in the Fourier spectrum.

We finally analyze the performance of the regularization strategy in the presence of noise. We use $16 \times 16$ lowresolution images to reconstruct a $64 \times 64$ high-resolution image. We consider the cases with no registration error, and with random registration errors of maximum 10 degrees with a uniform distribution. We further add a white gaussian noise with zero mean to the low resolution images, which results into a SNR of $30 \mathrm{~dB}$. Figure 11 shows the reconstruction results for both the Levenberg-Marquardt algorithm and the $\ell_{1}$ regularized solution. Again with both image and registration noise, the regularized solution rapidly converges to a high reconstruction quality and performs better than the LevenbergMarquardt algorithm. 


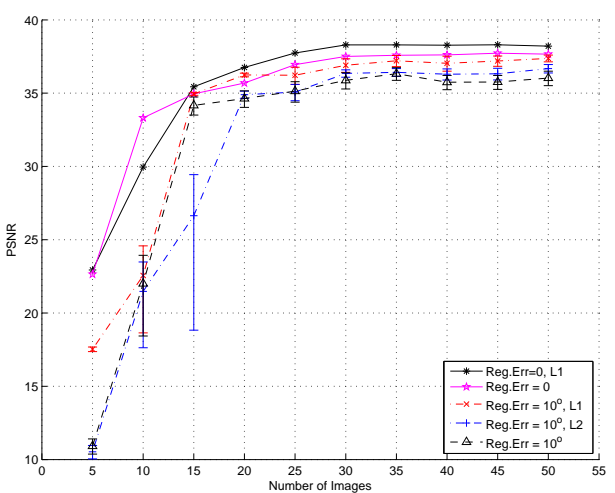

Fig. 10: Quality of the reconstruction of a 64 x 64 pixel image vs the number of $16 \times 16$ pixel images, for different regularization methods.

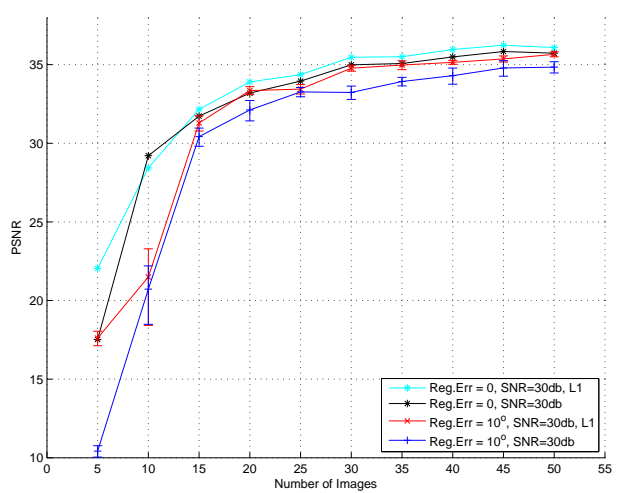

Fig. 11: Quality of the reconstruction of a 64 x 64 pixel image vs the number of $16 \times 16$ pixel images for the $\ell_{1}$-regularized algorithm in the presence of noise $(\mathrm{SNR}=30 \mathrm{~dB})$.

\section{Approximate solution}

We finally study in this section an approximate solution to the joint registration and super-resolution algorithms proposed in this paper. The estimation of the SFT coefficients using the full set of spherical harmonics is often constraining in practice due to memory requirements. An approximate solution can be obtained by successively computing subset of coefficients for blocks of spherical harmonics. The system matrix $\mathbf{S} \mathbf{R}$ contains spherical harmonics with different degrees and orders on columns. We propose an algorithm where the approximation is performed by partitioning the system matrix into smaller matrices $\mathbf{M}_{i}$ that have the same number of rows, but where the number of columns corresponds to different groups of spherical harmonics degrees. Since the spherical harmonics with same degree are correlated, we do not cut the matrix at arbitrary positions. Therefore, each submatrix $\mathbf{M}_{\mathbf{i}}$ has different number of columns, which is however always smaller than a predefined value that corresponds to the size of the largest block in the algorithm.

Let $\mathbf{a}_{\mathbf{i}}$ be the vector of coefficients corresponding to the spherical harmonics in $\mathbf{M}_{\mathbf{i}}$. We successively solve linear systems of equations of the form

$$
\mathbf{M}_{\mathbf{i}} \mathbf{a}_{\mathbf{i}}=\mathbf{z}_{\mathbf{i}}
$$

where $\mathbf{z}_{\mathbf{i}}$ is the residual which is calculated by

$$
\mathbf{z}_{\mathbf{i}}=\mathbf{z}_{\mathbf{i}-\mathbf{1}}-\mathbf{M}_{\mathbf{i}-\mathbf{1}} \mathbf{a}_{\mathbf{i}-\mathbf{1}}
$$

Note that initial residual $\mathbf{z}_{\mathbf{0}}$ is the image vector $\mathbf{z}$. Note that a similar decomposition scheme can be applied to the system in Eq. (25) in order to find the search direction. A small difference however resides in the order of the iterations as the convergence of the minimization is targeted in each block.

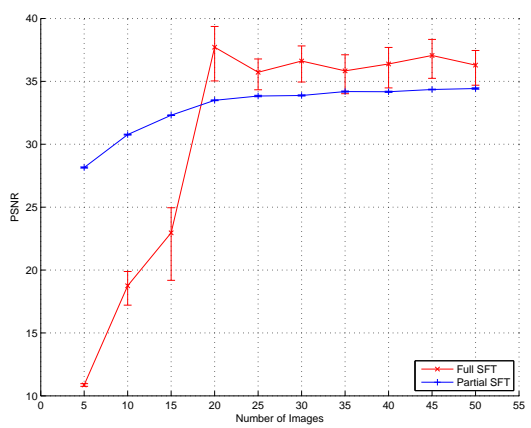

Fig. 12: Quality of the reconstruction of $120 \times 120$ images vs the number of $30 \times 30$ images for full and partial SFT implementation of the Levenberg-Marquardt algorithm. The low resolution images have a registration error of up to 10 degrees.

We now compare the performance of the approximate solution that we call 'Partial SFT' to the performance of the original Levenberg-Marquardt algorithms. Figure 13 shows the PSNR values for different number of images in case of partial and full SFTs implementations for the reconstruction of a 120x120 image from multiple 30x30 spherical images that have a random registration error of up to 10 degrees. We see that the approximate solution also converges with the increasing number of low resolution images, with a small penalty however compared to the original method. In addition, regrouping of low frequency spherical harmonics lets a better minimization when the number of images is small. Figure 12 shows two images reconstructed with the full and partial SFT implementation of the Levenberg-Marquardt algorithm. Although the quality is higher with the full SFT method around the edges, the quality of the partial SFT method stays comparable while the memory footprint is around 4 times smaller.

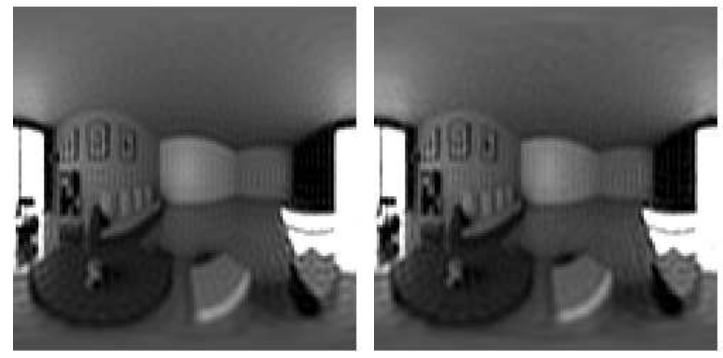

Fig. 13: Reconstruction of 120x 120 images from fifty $30 \times 30$ images with up to 10 degrees of registration errors for full(left) and partial (right) SFT methods. 
Lastly, we have tested our proposed algorithm with partial SFT approximation on real images. We captured 24 omnidirectional images with different rotations in 3D using a catadioptric camera. We only know coarsely the registration parameters. Figure 14 shows two low resolution omnidirectional images that have been mapped onto a sphere of 64 by 64 pixels. Such images are used for the reconstruction of higher resolution images of $128 \times 128$ pixels. Figure 15 shows the reconstructed images in the cases where the registration error has not been corrected, and where the joint registration and superresolution problem is solved with the Levenberg-Marquardt algorithm. It is clear that the second method provides higher reconstruction quality with sharper reconstruction thanks to the efficient correction of the registration errors.

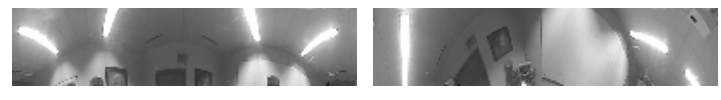

Fig. 14: Two of the low resolution omnidirectional images mapped onto sphere.
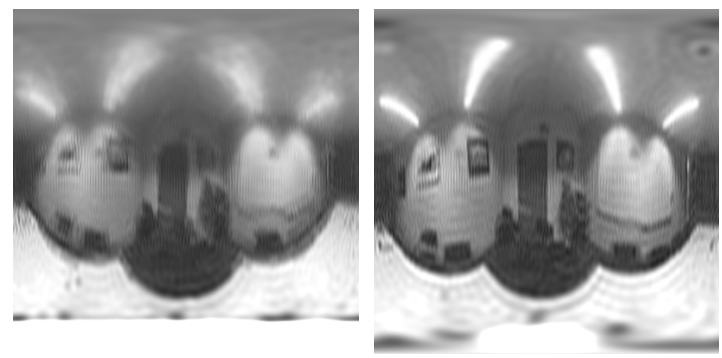

Fig. 15: Reconstructed full spherical images without correction of the registration error (Left) and with the joint registration and super-resolution with Levenberg-Marquardt algorithm (Right).

\section{CONCLUSIONS}

We have addressed the joint registration and superresolution problem from low resolution omnidirectional images with arbitrary rotations in $\mathrm{SO}(3)$. We have proposed an reconstruction algorithm based on a nonlinear least-squares norm optimization problem in the SFT domain, which is solved by a Levenberg-Marquardt method. An $\ell_{1}$-regularized solution has then been proposed to further improve the reconstruction quality or equivalently reduce the number of images that are necessary to achieve the target quality in the high resolution image. We have shown that the reconstruction methods are quite resilient to additive noise in the low resolution images, and that the solutions are independent of the sampling scheme used in the sensing device. Finally, we have proposed an approximate solution for the computation of the Fourier coefficients based on a partitioning of the problem. It provides close to exact solutions with much less memory requirements. The effective reconstruction performance in experiments with synthetic and natural omnidirectional images demonstrates the benefits of the proposed solution for super-resolution problems in omnivision applications with imperfect settings.

\section{APPENDIX}

A. Properties of $P_{m n}^{l}$

The functions $P_{m n}^{l}(\cos \beta)$ are the generalizations of the associated Legendre function and can be calculated relative to Jacobi polynomials [22] as

$P_{m n}^{l}(\cos \beta)=\sqrt{\left[\frac{(l-m) !(l+m) !}{(l-n) !(l+n) !}\right]} \sin ^{m-n} \frac{\beta}{2} \cos ^{m+n} \frac{\beta}{2} P_{l-m}^{(m-n, m+n)}(\cos \beta)$

They satisfy certain symmetry relations such as:

$$
\begin{array}{ll}
P_{m n}^{l}(x)=(-1)^{m+n} P_{n m}^{l}(x) & P_{m n}^{l}(x)=(-1)^{m-n} P_{-m,-n}^{l}(x) \\
P_{m n}^{l}(x)=P_{-n,-m}^{l}(x) & P_{m n}^{l}(x)=(-1)^{l+n} P_{-m, n}^{l}(-x)
\end{array}
$$

Jacobi Polynomial $P_{l-m}^{(m-n, m+n)}(\cos \beta)$ is given by

$$
\begin{aligned}
P_{l-m}^{(m-n, m+n)}(\cos \beta) & =\frac{\Gamma(l-n+1)}{(l-m) ! \Gamma(l+m+1)} \\
& \sum_{q=0}^{(l-m)}\left(\begin{array}{c}
l-m \\
q
\end{array}\right) \frac{\Gamma(l+m+q+1)}{\Gamma(m-n+q+1)}\left(\frac{\cos \beta-1}{2}\right)^{q}
\end{aligned}
$$

where $\Gamma(z)$ is the gamma function. For positive integers, the gamma function can be calculated as

$$
\Gamma(z)=(z-1) \text { ! }
$$

From $|m|<l,|n|<l$ and the symmetry properties in Eq. (32), we can assure that inputs to the Gamma functions in Eq. (33) are positive integers. Thus, Eq. (33) turns into

$$
\begin{aligned}
P_{l-m}^{(m-n, m+n)}(\cos \beta) & =\frac{(l-n) !}{(l-m) !(l+m) !} \\
& \sum_{q=0}^{(l-m)}\left(\begin{array}{c}
l-m \\
q
\end{array}\right) \frac{(l+m+q) !}{(m-n+q) !}\left(\frac{\cos \beta-1}{2}\right)^{q}
\end{aligned}
$$

\section{REFERENCES}

[1] C. Segall, R. Molina, and A. Katsaggelos, "High-resolution images from low-resolution compressed video," Signal Processing Magazine, IEEE, vol. 20 , no. 3, pp. 37- 48, 2003.

[2] S. Farsiu, D. Robinson, M. Elad, and P. Milanfar, "Advances and challenges in super-resolution," International Journal of Imaging Systems and Technology, vol. 14, pp. 47-57, Jan 2004.

[3] S. C. Park, M. K. Park, and M. G. Kang, "Super-resolution image reconstruction: a technical overview," Signal Processing Magazine, IEEE, vol. 20, no. 3, pp. 21- 36, 2003.

[4] G. Costa and J. Bermudez, "Registration errors: Are they always bad for super-resolution?," Signal Processing, IEEE Transactions on, vol. 57, no. 10, pp. 3815-3826, 2009.

[5] P. Vandewalle, L. Sbaiz, J. Vandewalle, and M. Vetterli, "Superresolution from unregistered and totally aliased signals using subspace methods," IEEE Trans. Signal Processing, vol. 55, pp. 3687-3703, 2007.

[6] M. Ng, J. Koo, and N. Bose, "Constrained Total Least-Squares Computations for High-Resolution Image Reconstruction with Multisensors," sensors, vol. 50, pp. 35-42, 2002.

[7] Y. He, K. Yap, L. Chen, and L. Chau, "A Nonlinear Least Square Technique for Simultaneous Image Registration and Super-Resolution," Image Processing, IEEE Transactions on, vol. 16, no. 11, pp. 28302841, 2007.

[8] K.-H. Yap, Y. He, Y. Tian, and L.-P. Chau, "A nonlinear $l_{1}$ norm approach for joint image registration and super-resolution," Signal Processing Letters, IEEE, vol. 16, no. 11, pp. 981-984, 2009.

[9] H. Kawasaki, K. Ikeuchi, and M. Sakauchi, "Super-resolution Omnidirectional Camera Images Using Spatio-temporal Analysis," Electronics and Communications in Japan Part 3 Fundamental Electronic Science, vol. 89 , no. 6 , p. $47,2006$.

[10] H. Nagahara, Y. Yagi, and M. Yachida, "Super-resolution from an omnidirectional image sequence," Industrial Electronics Society, 2000. IECON 2000. 26th Annual Confjerence of the IEEE, vol. 4, 2000. 
[11] B. Rafaely and M. Park, "Super-resolution spherical microphone arrays," in Electrical and Electronics Engineers in Israel, 2004. Proceedings. 2004 23rd IEEE Convention of, pp. 424-427, 2004.

[12] Z. Fan and Z. Qi-dan;, "Super-resolution image reconstruction for omnivision based on pocs," Control and Decision Conference, 2009. CCDC '09. Chinese, pp. 5045 - 5049, Jun 2009.

[13] C. Geyer and K. Daniilidis, "Catadioptric Projective Geometry," International Journal of Computer Vision, vol. 45, no. 3, pp. 223-243, 2001.

[14] Z. Arican and P. Frossard, "Super-resolution from unregistered omnidirectional images," in Proceedings of the Int. Conf. on Pattern Recognition, 2008.

[15] Z. Arican and P. Frossard, "11 regularized super-resolution from unregistered omnidirectional images," in Proceedings of IEEE ICASSP, 2009.

[16] K. Daniilidis, A. Makadia, and T. Bulow, "Image processing in catadioptric planes: spatiotemporal derivatives and optical flow computation," Omnidirectional Vision, 2002. Proceedings. Third Workshop on, pp. 310, 2002.

[17] S. Baker and S. Nayar, "A theory of single-viewpoint catadioptric image formation," Int J Comput Vis, vol. 35, pp. 175-196, Jan 1999.

[18] J. Driscoll and D. Healy, "Computing fourier transforms and convolutions on the 2-sphere," Advances in Applied Mathematics, vol. 15, pp. 202-250, Jan 1994.

[19] J.R. Driscoll and D.M. Healy, Jr., "Asymptotically fast algorithms for spherical and related transforms," 30th Annual Symposium on Foundations of Computer Science, pp. 344 - 349, October 1989.

[20] D. Healy, D. Rockmore, P. Kostelec, and S. Moore, "FFTs for the 2sphere - improvements and variations," Journal of Fourier Analysis and Applications, vol. 9, no. 4, pp. 341-385, 2003.

[21] I. Tosic and P. Frossard, "FST-based Reconstruction of 3D-models from Non-Uniformly Sampled Datasets on the Sphere," Picture Coding Symposium, 2006.

[22] G. Chirikjian and A. Kyatkin, Engineering Applications of Noncommutative Harmonic Analysis: With Emphasis on Rotation and Motion Groups. CRC Press, 2000.

[23] D. W. Marquardt, "An algorithm for least-squares estimation of nonlinear parameters," SIAM Journal on Applied Mathematics, vol. 11, no. 2, pp. $431-441,1963$

[24] S.-J. Kim, K. Koh, M. Lustig, S. Boyd, and D. Gorinevsky, "An interiorpoint method for large-scale 11-regularized least squares," Selected Topics in Signal Processing, IEEE Journal of, vol. 1, pp. 606-617, Dec. 2007. 